transplantation. Murine models of acute liver injury are characterised by changes in the hepatic macrophage compartment, with an initial accumulation of pro-inflammatory Ly6 $\mathrm{C}^{\text {hi }}$ monocytes and loss of Kupffer cells, followed by the dominance of recruited pro-reparative Ly6 $\mathrm{C}^{\text {lo }}$ macrophages. Current experimental immunomodulatory therapeutics like MC21 and Cenicriviroc inhibit Ly6 $\mathrm{C}^{\text {hi }}$ monocyte recruitment. In non-hepatic sterile injury models IL-4 is known to promote pro-reparative macrophage functions and IL-4 administered prior to injury with carbon tetrachloride $\left(\mathrm{CCl}_{4}\right)$ has been shown to be hepatoprotective by directly promoting hepatocyte proliferation. We therefore aimed to assess the potential pro-reparative and immunomodulatory effects of therapeutic IL-4 administered following acute liver injury with $\mathrm{CCl}_{4}$.

Methods Male C57Bl/6 mice were given $\mathrm{CCl}_{4}$ intraperitoneally to induce an acute liver injury. IL-4 was administered in the form of an immune complex subcutaneously. To investigate the role of IL-4R $\alpha$ signalling in bone marrow derived cells, whole-body and tissue-protected chimeras were generated with wild type or IL-4R ${ }^{-/-}$donor bone marrow. Results were analysed using immunohistochemistry of tissue sections, serum biochemistry and flow cytometric analysis of leukocytes.

Results Therapeutic administration of IL-4 following $\mathrm{CCl}_{4}$ reduced markers of hepatic injury (ALT and necrotic area) and enhanced hepatic regeneration as measured by hepatocyte proliferation. This was paralleled by profound alterations to the monocyte/macrophage pool, with increases in the number of pro-reparative Ly6C $\mathrm{C}^{\mathrm{lo}}$ macrophages but also a significant reduction in the number of pro-inflammatory hepatic Ly $6 \mathrm{C}^{\text {hi }}$ monocytes. Using chimeras, we have shown that $\mathrm{Ly}_{6 \mathrm{C}} \mathrm{lo}^{\mathrm{m}} \mathrm{mac}-$ rophage accumulation required cell-intrinsic, IL-4R $\alpha$-dependent proliferation and the loss of hepatic Ly6 $\mathrm{C}^{\text {hi }}$ monocytes was dependent on cell-intrinsic, IL-4R $\alpha$ signalling. Importantly, analysis of kidney, spleen and blood revealed that the loss of Ly6 $\mathrm{C}^{\text {hi }}$ monocytes secondary to IL-4 treatment was not limited to the liver and occurred systemically. In vitro and ex vivo assays showed that the reduction in $\mathrm{Ly} 6 \mathrm{C}^{\text {hi }}$ monocytes in response to administration of IL-4 was due to IL-4R $\alpha$-dependent apoptosis of circulating monocytes rather than decreased output from the bone marrow.

Conclusion This novel role of IL-4 offers potential therapeutic benefits over monocyte inhibitors by not only reducing proinflammatory Ly $6 \mathrm{C}^{\text {hi }}$ monocyte numbers through apoptosis but also increasing the number of pro-reparative Ly6 $\mathrm{C}^{\text {lo }}$ macrophages.

\section{OTU-08 HEAL (HIV NON-VIRAL LIVER DISEASE) STUDY: THE ROLE OF ALCOHOL, METABOLIC SYNDROME OR HIV ITSELF?}

${ }^{1}$ Harriet Sharp*, 'Dev Katarey, 'Laura Vickers, ${ }^{1,2}{ }^{2}$ vonne Gilleece, ${ }^{1,2}$ Sumitra Verma. ${ }^{1}$ Brighton and Sussex University Hospital NHS Trust, Brighton, UK; ${ }^{2}$ Brighton and Sussex Medical School, Brighton, UK

\subsection{6/gutjnl-2019-BSGAbstracts.199}

Introduction In view of advances in viral hepatitis treatment, future chronic liver disease (CLD) in people living with HIV (PLWH) is likely to be due to non-viral hepatitis aetiologies. Potential contributors include alcohol, metabolic syndrome (MS) or antiretrovirals (ARV). Understanding risk factors and early recognition of CLD is essential to prevent long-term morbidity and mortality. This prospective cohort study investigates the prevalence and predictors of CLD in PLWH with abnormal liver function.

Methods Inclusion criteria were PLWH, negative viral hepatitis serology and elevated transaminases over 6 months. Consenting individuals completed an Alcohol Use Disorders Identification Test (AUDIT), underwent MS assessment and transient elastography. Study definitions: significant hepatic steatosis (SHS) - controlled attenuation parameter (CAP) $\geq 237 \mathrm{~dB} / \mathrm{m}$; significant hepatic fibrosis (SHF) and cirrhosis - liver stiffness measurement $\geq 7.1 \mathrm{kPa}$ and $\geq 11.5 \mathrm{kPa}$ respectively. SHF risk factors consisted of MS, hazardous drinking (AUDIT $\geq 8$ ) and hepatotoxic ARV use.

Results Of 429 eligible individuals, 219 have been recruited. Mean age was $52.3 \pm 9.8$ years, $92.2 \%$ were male, $96.3 \%$ with undetectable viral load, mean HIV duration of $15.8 \pm 7.6$ years. Overall prevalence of SHS was 63\% $(n=137)$, and SHF was $21 \%(n=47)$, of whom $35(74 \%)$ had SHS and $17(36 \%)$ had cirrhosis.

On binary logistic regression, HDL and AUDIT score were significantly associated with SHF whereas CD4 baseline, HIV duration, BMI, hypertension, diabetes, and duration on ARV were not. Predictors of SHS included BMI, HDL and AUDIT score.

No classical risk factors were identified in $8(17 \%)$ individuals with SHF but they had significantly shorter HIV duration and higher peak ALT compared to those with risk factors for SHF (table 1).

Abstract 0TU-08 Table 1 Characteristics of patients with SHF

\begin{tabular}{llll}
\hline & No risk factors $\mathrm{n}=8$ & Risk factors $\mathrm{n}=34$ & Significance \\
\hline Age (years) & $45.5 \pm 9.6$ & $54.6 \pm 8.3$ & $\mathrm{P}=0.069$ \\
HIV duration (years) & $9.1 \pm 4.5$ & $17.0 \pm 8.4$ & $\mathrm{P}=0.006^{*}$ \\
Baseline CD4 (10 $/ \mathrm{L})$ & $421 \pm 240$ & $546 \pm 1372$ & $\mathrm{P}=0.407$ \\
ALT peak (iu/L) & $112.6 \pm 79.6$ & $66.8 \pm 31.2$ & $\mathrm{P}=0.048^{*}$ \\
\hline
\end{tabular}

Conclusion There is considerable liver disease burden in PLWH with elevated transaminases, with nearly $2 / 3$ having SHS and 1/5 SHF. MS risk factors and alcohol use appear to predict both SHS and SHF. However, one fifth of individuals with SHF have no identifiable risk factors, raising the real possibility of immune dysregulation or direct hepatotoxicity of HIV in this subpopulation. Our data highlights the need to implement screening strategies for CLD in PLWH alone to ensure timely Hepatology input, and the importance of providing appropriate advice regarding alcohol intake and weight loss.

\section{OTU-09 USE OF EARLY-TIPSS IN PATIENTS WITH OESOPHAGEAL VARICEAL BLEEDING, A UK DUAL-CENTRE RANDOMISED CONTROL TRIAL}

${ }^{1}$ Philip Dunne*, ${ }^{1}$ Rohit Sinha, ${ }^{2}$ Adrian Stanley, ${ }^{2}$ Neil Lachlan, ${ }^{1}$ Hamish Ireland, ${ }^{1}$ Aman Shams, ${ }^{1}$ Peter Hayes. ${ }^{1}$ Royal Infirmary Edinburgh, Edinburgh, UK; ${ }^{2}$ Glasgow Royal Infirmary, Glasgow, UK

\subsection{6/gutjnl-2019-BSGAbstracts.200}

Introduction International guidelines recommend consideration of early-TIPSS for high-risk patients (Childs-Pugh grade 


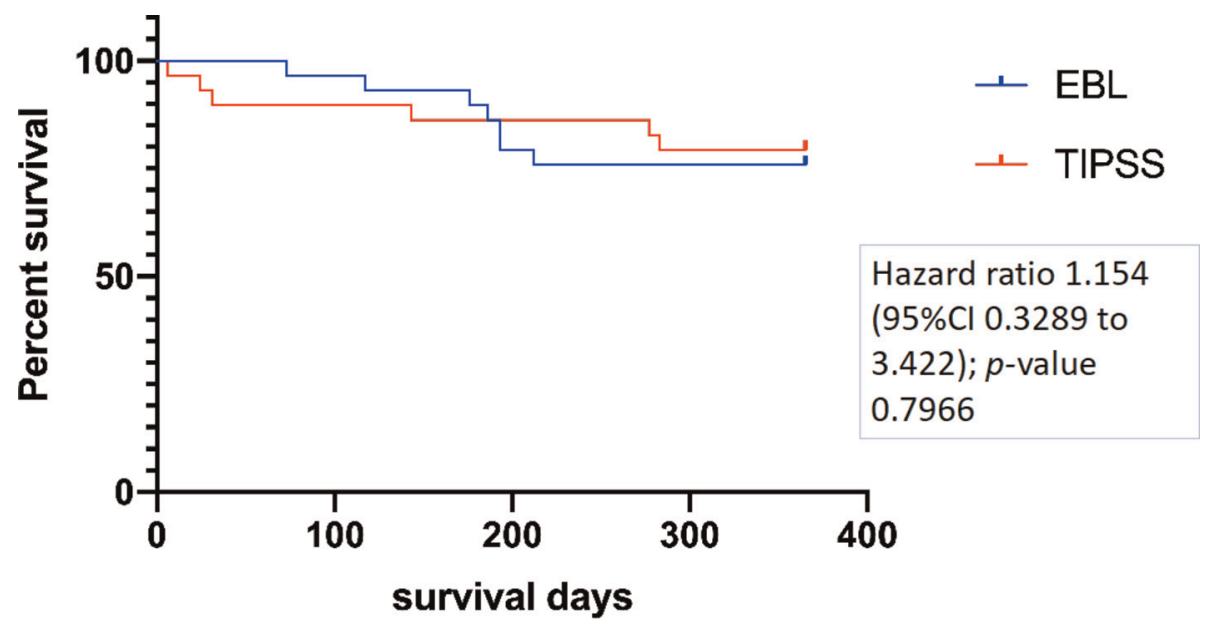

Abstract OTU-09 Figure 1

$\mathrm{B}$ and C) presenting with oesophageal variceal bleeding (VB), following initial haemostasis with endotherapy and drugs. However, this is based on a single randomised controlled trial (RCT).

Methods An open-label, parallel-group, RCT was performed at 2 experienced UK centres. Cirrhotic patients with a Childs Pugh (CP) score $\geq 8$ presenting to either centre with VB were considered for inclusion in the study. Following haemostasis with endoscopic band ligation (EBL) and vasoactive drugs, consenting participants were randomised to either continued EBL \pm non-selective beta-blockers (standard care) or earlyTIPSS (ie within 72hrs). All patients were followed up for 1 year, or until death. The primary endpoint was survival at 1 year. Secondary endpoints included early and late re-bleeding ( $<6$ weeks and 6 weeks -1 year). The study was completed between April 2012 - January 2018.

Results 216 patients were screened with 58 eligible participants ultimately included (29 per group). EBL and early-TIPSS were well matched for age (53.9 vs 49.4); CP score (10.1 vs 9.8); and MELD score (16.8 vs 16.4 ) respectively.

On intention to treat, 1-year survival rates were $75.8 \%$ in the EBL group versus $79.3 \%$ in the early-TIPSS group $(p=0.79)$ (see figure 1). 1 patient in the EBL group received TIPSS as rescue therapy, but later died. 4 patients who were randomised to early-TIPSS did not receive this intervention due to time delays and therefore underwent standard care. However, on per-protocol analysis, no survival benefit was observed between groups $(\mathrm{p}=0.85)$. In patients with ChildsPugh C cirrhosis $(n=33)$ no difference in survival was seen between groups $(p=0.81)$. At 6 weeks, rebleeding occurred in 2 patients in both the EBL and early-TIPSS groups. At 1-year, rebleeding occurred in 8 patients in the EBL group compared with 6 patients in the early-TIPSS group. No significant differences in rates of rebleeding were seen at the specified time points. Additionally, no significant differences were observed between the two treatment groups with respect to serious adverse events.

Conclusions Contrary to the previous study, our findings do not support the use of early-TIPSS in the reduction of mortality or rebleeding in patients with cirrhosis who are hospitalised for acute variceal bleeding. Given the challenges of resourcing the widespread provision of early-TIPSS, further multi-centred studies with larger patient numbers are required to resolve this important issue.

\section{OTU-10 IMPACT OF CIRRHOSIS SEVERITY ON CLINICAL OUTCOMES IN HEPATOCELLULAR CARCINOMA IN ENGLAND}

${ }^{1}$ Robert Driver*, ${ }^{2}$ Anya Burton, ${ }^{1}$ Amy Downing, ${ }^{3}$ Aileen Marshall, ${ }^{1}$ Eva Morris, ${ }^{1}$ lan Rowe. ${ }^{1}$ University of Leeds, Leeds, UK; ${ }^{2}$ Public Health England, Bristol, UK; ${ }^{3}$ HCC-UK Steering Group, UK

\subsection{6/gutjnl-2019-BSGAbstracts.201}

Background Survival in hepatocellular carcinoma (HCC) is determined by both cancer stage and severity of underlying liver disease. International guidelines based on data from clinical trials provide a benchmark for survival following different HCC treatments [1]. The aim of this study was to estimate the impact of cirrhosis severity on overall survival for patients with HCC and cirrhosis in England.

Methods All patients registered with a new diagnosis of HCC between 2007 and 2016 in England were identified from the National Cancer Registration and Analysis Service records, which are linked to the Hospital Episode Statistics dataset. These records were used to identify patients with cirrhosis, their baseline characteristics and HCC treatments received. Cirrhosis severity at HCC diagnosis was categorised by the Baveno IV consensus: compensated cirrhosis defined by stage 1 (no ascites or varices) and stage 2 (non-bleeding varices), and decompensated cirrhosis defined by stage 3 (ascites) or stage 4 (bleeding varices).

Following HCC treatment, the linked record was analysed to identify subsequent decompensation events during 5 years of follow-up. Competing risk models were used to describe the risk of death after decompensation ('death due to liver failure') and the risk of death without decompensation ('death due to HCC').

Results Among 19,436 patients registered with HCC, 11,337 were identified with cirrhosis and were included in the analyses. The majority of patients $(6,207,54.7 \%)$ were ineligible for specific HCC treatment and received best supportive care. The most common HCC treatments were ablation $(1,228$ patients, 10.8\%) and trans-arterial chemoembolization (TACE; 2,021 patients, $17.8 \%$ ).

Among those who received ablation, the median survival was 41.9 months in those without varices at HCC diagnosis, 33.8 months in those with non-bleeding varices, and 23.9 months in those with previous decompensation $(\mathrm{P}<0.001)$. Among those who received TACE, median overall survival in 